Algebraic 85 Geometric Topology

Volume 3 (2003) 1277-1289

Published: 31 December 2003

ATG

\title{
Cell-like resolutions preserving cohomological dimensions
}

\author{
Michael Levin
}

\begin{abstract}
We prove that for every compactum $X$ with $\operatorname{dim}_{\mathbf{Z}} X \leq n \geq 2$ there is a cell-like resolution $r: Z \longrightarrow X$ from a compactum $Z$ onto $X$ such that $\operatorname{dim} Z \leq n$ and for every integer $k$ and every abelian group $G$ such that $\operatorname{dim}_{G} X \leq k \geq 2$ we have $\operatorname{dim}_{G} Z \leq k$. The latter property implies that for every simply connected $\mathrm{CW}$-complex $K$ such that e-dim $X \leq K$ we also have e-dim $Z \leq K$.
\end{abstract}

AMS Classification 55M10, 54F 45

Keywords Cohomological dimension, cell-like resolution

\section{Introduction}

A space $X$ is always assumed to be separable metrizable. The cohomological dimension $\operatorname{dim}_{G} X$ of $X$ with respect to an abelian group $G$ is the least number $n$ such that $\check{H}^{n+1}(X, A ; G)=0$ for every closed subset $A$ of $X$. It was known long ago that $\operatorname{dim} X=\operatorname{dim}_{\mathbf{Z}} X$ if $X$ is finite dimensional. The first example of an infinite dimensional compactum (=compact metric space) with finite integral cohomological dimension was constructed by Dranishnikov [2] in 1987. In 1978 Edwards $[10,16]$ discovered his celebrated resolution theorem revealing a close relation between $\operatorname{dim}_{\mathbf{Z}}$ and $\operatorname{dim}$. The Edwards resolution theorem says that a compactum of $\operatorname{dim}_{\mathbf{Z}} \leq n$ can be obtained as the image of a cell-like map defined on a compactum of $\operatorname{dim} \leq n$. A compactum $X$ is cell-like if any map $f: X \longrightarrow K$ from $X$ to a $\mathrm{CW}$-complex $K$ is null-homotopic. A map is cell-like if its fibers are cell-like. The reduced Čech cohomology groups of a cell-like compactum are trivial with respect to any group $G$.

The Edwards resolution theorem addresses only the case of integral cohomological dimension. It seems natural to look for generalizations of this theorem taking into consideration other abelian groups. Indeed, such an investigation has been of considerable interest in cohomological dimension theory. It mainly went in two directions. 
The first one is to adjust resolutions for a given group $G$ replacing cell-like maps by $G$-acyclic maps. A map is $G$-acyclic if the reduced Cech cohomology groups modulo $G$ of the fibers are trivial. By the Vietoris-Begle theorem a $G$-acyclic map cannot raise the cohomological dimension $\operatorname{dim}_{G}$. Let us give two examples of results of this type.

Theorem 1.1 [3] Let $p$ be a prime number and let $X$ be a compactum with $\operatorname{dim}_{\mathbf{Z}_{p}} X \leq n$. Then there are a compactum $Z$ with $\operatorname{dim} Z \leq n$ and a $\mathbf{Z}_{p}$-acyclic map $r: Z \longrightarrow X$ from $Z$ onto $X$.

Theorem 1.2 [15] Let $G$ be an abelian group and let $X$ be a compactum with $\operatorname{dim}_{G} X \leq n, n \geq 2$. Then there are a compactum $Z$ with $\operatorname{dim}_{G} Z \leq n$ and $\operatorname{dim} Z \leq n+1$ and a $G$-acyclic map $r: Z \longrightarrow X$ from $Z$ onto $X$.

The other direction of investigation is to construct cell-like resolutions preserving cohomological dimensions with respect to several abelian groups. Below are some results of this type.

Theorem 1.3 [4] Let $p$ be a prime number and let a compactum $X$ be such that $\operatorname{dim}_{\mathbf{Z}_{p}} X \leq n$ and $\operatorname{dim}_{\mathbf{Z}[1 / p]} X \leq n, n \geq 2$. Then there are a compactum $Z$ with $\operatorname{dim} Z \leq n+1, \operatorname{dim}_{\mathbf{Z}_{p}} Z \leq n$ and $\operatorname{dim}_{\mathbf{Z}[1 / p]} Z \leq n$ and a cell-like map $r: Z \longrightarrow X$ from $Z$ onto $X$.

Theorem 1.4 [6] Let $\mathcal{L}$ be a subset of the set of primes and let $X$ be a compactum such that $\operatorname{dim}_{\mathbf{Z}} X \leq n$ and $\operatorname{dim}_{\mathbf{Z}_{p}} X \leq k, n<2 k-1$ for every $p \in \mathcal{L}$. Then there are a compactum $Z$ with $\operatorname{dim} Z \leq n$ and $\operatorname{dim}_{\mathbf{Z}_{p}} Z \leq k$ for every $p \in \mathcal{L}$ and a cell-like map $r: Z \longrightarrow X$ from $Z$ onto $X$.

Theorem 1.5 [13] Let $p, q$ be distinct prime numbers and let $n$ be an integer $>1$. Then for a compactum $X$ with $\operatorname{dim}_{\mathbf{Z}_{p}} X \leq n, \operatorname{dim}_{\mathbf{Z}_{(q)}} X \leq n$ and $\operatorname{dim}_{\mathbf{Z}} X \leq n+1$ there exist an $(n+1)$-dimensional compactum $Z$ with $\operatorname{dim}_{\mathbf{Z}_{p}} Z \leq n, \operatorname{dim}_{\mathbf{Z}_{(q)}} Z \leq n$ and a cell-like map $r: Z \longrightarrow X$ from $Z$ onto $X$.

This paper goes along the line of investigation represented by Theorems 1.3, 1.4 and 1.5. These theorems can be regarded as particular cases of the following general problem: Let $X$ be a compactum with $\operatorname{dim}_{\mathbf{Z}} X \leq n$. Do there exist an $n$-dimensional compactum $Z$ and a cell-like map from $Z$ onto $X$ such that $\operatorname{dim}_{G} Z \leq \operatorname{dim}_{G} X$ for every abelian group $G$ ? The goal of this paper is to answer this problem affirmatively in cohomological dimensions larger than 1 . Namely we will prove the following theorem. 
Theorem 1.6 Let $X$ be a compactum with $\operatorname{dim}_{\mathbf{Z}} X \leq n \geq 2$. Then there exist a compactum $Z$ with $\operatorname{dim} Z \leq n$ and a cell-like map $r: Z \longrightarrow X$ from $Z$ onto $X$ such that for every integer $k \geq 2$ and every group $G$ such that $\operatorname{dim}_{G} X \leq k$ we have $\operatorname{dim}_{G} Z \leq k$.

Theorem 1.6 can be reformulated in terms of extensional dimension $[7,8]$. The extensional dimension of $X$ is said not to exceed a CW-complex $K$, written e- $\operatorname{dim} X \leq K$, if for every closed subset $A$ of $X$ and every map $f: A \longrightarrow K$ there is an extension of $f$ over $X$. It is well-known that $\operatorname{dim} X \leq n$ is equivalent to e-dim $X \leq \mathbf{S}^{n}$ and $\operatorname{dim}_{G} X \leq n$ is equivalent to e-dim $X \leq K(G, n)$ where $K(G, n)$ is an Eilenberg-Mac Lane complex of type $(G, n)$. The following theorem shows a close connection between cohomological and extensional dimensions.

Theorem 1.7 [5] Let $X$ be a compactum and let $K$ be a simply connected $C W$-complex. Consider the following conditions:

(1) $\quad$ e- $\operatorname{dim} X \leq K$;

(2) $\operatorname{dim}_{H_{i}(K)} X \leq i$ for every $i>1$;

(3) $\operatorname{dim}_{\pi_{i}(K)} X \leq i$ for every $i>1$.

Then (2) and (3) are equivalent and (1) implies both (2) and (3). If $X$ is finite dimensional then all the conditions are equivalent.

Theorems 1.6 and 1.7 imply the following:

Theorem 1.8 Let $X$ be a compactum with $\operatorname{dim}_{\mathbf{Z}} X \leq n \geq 2$. Then there exist a compactum $Z$ with $\operatorname{dim} Z \leq n$ and a cell-like map $r: Z \longrightarrow X$ from $Z$ onto $X$ such that for every simply connected $C W$-complex $K$ such that e- $\operatorname{dim} X \leq K$ we have e- $\operatorname{dim} Z \leq K$.

Proof Let $Z$ and $r: Z \longrightarrow X$ be as in Theorem 1.6. Let a simply connected CW-complex $K$ be such that e- $\operatorname{dim} X \leq K$. Then by Theorem 1.7, $\operatorname{dim}_{H_{i}(K)} X \leq i$ for every $i>1$ and hence by Theorem 1.6, $\operatorname{dim}_{H_{i}(K)} Z \leq i$ for every $i>1$. Then since $Z$ is finite dimensional it follows from Theorem 1.7 that $\mathrm{e}-\operatorname{dim} Z \leq K$.

Note that the restriction $k \geq 2$ in Theorem 1.6 cannot be omitted. Indeed, take an infinite dimensional compactum $X$ with $\operatorname{dim}_{\mathbf{Q}} X=1$ and $\operatorname{dim}_{\mathbf{Z}} X=2$ (such a compactum was constructed by Dydak and Walsh [12]) and let $r: Z \longrightarrow X$ be a cell-like map of a 2 -dimensional compactum $Z$ onto $X$. Then $\operatorname{dim}_{\mathbf{Q}} Z=2$ 
since otherwise by a result of Daverman [1] we would have $\operatorname{dim} X \leq 2$. This observation also shows that Theorem 1.8 does not hold for non-simply connected complexes $K$.

The author would like to thank the referee for the careful reading of the paper and valuable suggestions.

\section{Preliminaries}

A map between $\mathrm{CW}$-complexes is said to be combinatorial if the preimage of every subcomplex of the range is a subcomplex of the domain.

Let $M$ be a simplicial complex and let $M^{[k]}$ be the $k$-skeleton of $M$ (=the union of all simplexes of $M$ of $\operatorname{dim} \leq k)$. By a resolution $E W(M, k)$ of $M$ we mean a CW-complex $E W(M, k)$ and a combinatorial map $\omega: E W(M, k) \longrightarrow M$ such that $\omega$ is 1-to-1 over $M^{[k]}$. Let $f: N \longrightarrow K$ be a map of a subcomplex $N$ of $M$ into a CW-complex $K$. The resolution is said to be suitable for $f$ if the map $\left.f \circ \omega\right|_{\omega^{-1}(N)}$ extends to a map $f^{\prime}: E W(M, k) \longrightarrow K$. We will call $f^{\prime}$ a resolving map for $f$. The resolution is said to be suitable for a compactum $X$ if for every simplex $\Delta$ of $M$, e-dim $X \leq \omega^{-1}(\Delta)$. Note that if $\omega: E W(M, k) \longrightarrow M$ is a resolution suitable for $X$ then for every map $\phi: X \longrightarrow M$ there is a map $\psi: X \longrightarrow E W(M, k)$ such that for every simplex $\Delta$ of $M,(\omega \circ \psi)\left(\phi^{-1}(\Delta)\right) \subset \Delta$. We will call $\psi$ a combinatorial lifting of $\phi$.

Let $M$ be a finite simplicial complex. Let $f: N \longrightarrow K$ be a cellular map from a subcomplex $N$ of $M$ to a CW-complex $K$ such that $M^{[k]} \subset N$. Following $[14,15]$ we will construct a resolution $\omega: E W(M, k) \longrightarrow M$ of $M$ which is suitable for $f$. In the sequel we will refer to this resolution as the standard resolution for $f$. We will associate with the standard resolution a cellular resolving map $f^{\prime}: E W(M, k) \longrightarrow K$ which will be called the standard resolving map. The standard resolution is constructed by induction on $n=\operatorname{dim}(M \backslash N)$.

For $M=N$ set $E W(M, k)=M$ and let $\omega: E W(M, k) \longrightarrow M$ be the identity map with the standard resolving map $f^{\prime}=f$. Let $n>k$. Denote $M^{\prime}=N \cup M^{[n-1]}$ and assume that $\omega^{\prime}: E W\left(M^{\prime}, k\right) \longrightarrow M^{\prime}$ is the standard resolution of $M^{\prime}$ for $f$ with the standard resolving map $f^{\prime}: E W\left(M^{\prime}, k\right) \longrightarrow K$. The standard resolution $\omega: E W(M, k) \longrightarrow M$ is constructed as follows.

The CW-complex $E W(M, k)$ is obtained from $E W\left(M^{\prime}, k\right)$ by attaching the mapping cylinder of $\left.f^{\prime}\right|_{\omega^{\prime-1}(\partial \Delta)}$ to $\omega^{\prime-1}(\partial \Delta)$ for every $n$-simplex $\Delta$ of $M$ which is not contained in $M^{\prime}$. Let $\omega: E W(M, k) \longrightarrow M$ be the projection which 
extends $\omega^{\prime}$ by sending each mapping cylinder to the corresponding $n$-simplex $\Delta$ such that the $K$-part of the cylinder is sent to the barycenter of $\Delta$ and each interval connecting a point of $\omega^{\prime-1}(\partial \Delta)$ with the corresponding point of the $K$ part of the cylinder is sent linearly to the interval connecting the corresponding point of $\partial \Delta$ with the barycenter of $\Delta$. We can naturally define the extension of $\left.f^{\prime}\right|_{\omega^{\prime-1}(\partial \Delta)}$ over its mapping cylinder by sending each interval of the cylinder to the corresponding point of $K$. Thus we define the standard resolving map which extends $f^{\prime}$ over $E W(M, k)$. The $\mathrm{CW}$-structure of $E W(M, k)$ is induced by the $\mathrm{CW}$-structure of $E W\left(M^{\prime}, k\right)$ and the natural $\mathrm{CW}$-structures of the mapping cylinders in $E W(M, k)$. Then with respect to this $\mathrm{CW}$-structure the standard resolving map is cellular and $\omega$ is combinatorial.

From the construction of the standard resolution it follows that for each simplex $\Delta$ of $M, \omega^{-1}(\Delta)$ is either contractible or homotopy equivalent to $K$ and for every $x \in M, \omega^{-1}(x)$ is either a singleton or homeomorphic to $K$. It is easy to check that if $M$ and $K$ are $(k-1)$-connected then so is $E W(M, k)$. Also note that for every subcomplex $T$ of $M,\left.\omega\right|_{\omega^{-1}(T)}: E W(T, k)=\omega^{-1}(T) \longrightarrow T$ is the standard resolution of $T$ for $\left.f\right|_{N \cap T}$.

All groups are assumed to be abelian and functions between groups are homomorphisms. $\mathcal{P}$ stands for the set of primes. For a non-empty subset $\mathcal{A}$ of $\mathcal{P}$ let $S(\mathcal{A})=\left\{p_{1}^{n_{1}} p_{2}^{n_{2}} \ldots p_{k}^{n_{k}}: p_{i} \in \mathcal{A}, n_{i} \geq 0\right\}$ be the set of positive integers with prime factors from $\mathcal{A}$ and for the empty set define $S(\emptyset)=\{1\}$. Let $G$ be a group and $g \in G$. We say that $g$ is $\mathcal{A}$-torsion if there is $n \in S(\mathcal{A})$ such that $n g=0$ and $g$ is $\mathcal{A}$-divisible if for every $n \in S(\mathcal{A})$ there is $h \in G$ such that $n h=g$. $\operatorname{Tor}_{\mathcal{A}} G$ is the subgroup of the $\mathcal{A}$-torsion elements of $G$. $G$ is $\mathcal{A}$-torsion if $G=\operatorname{Tor}_{\mathcal{A}} G$, $G$ is $\mathcal{A}$-torsion free if $\operatorname{Tor}_{\mathcal{A}} G=0$ and $G$ is $\mathcal{A}$-divisible if every element of $G$ is $\mathcal{A}$-divisible.

$G$ is $\mathcal{A}$-local if $G$ is $(\mathcal{P} \backslash \mathcal{A})$-divisible and $(\mathcal{P} \backslash \mathcal{A})$-torsion free. The $\mathcal{A}$ localization of $G$ is the homomorphism $G \longrightarrow G \otimes \mathbf{Z}_{(\mathcal{A})}$ defined by $g \longrightarrow g \otimes 1$ where $\mathbf{Z}_{(\mathcal{A})}=\{n / m: n \in \mathbf{Z}, m \in S(\mathcal{P} \backslash \mathcal{A})\} . \quad G$ is $\mathcal{A}$-local if and only if the $\mathcal{A}$-localization of $G$ is an isomorphism. A map between two simply connected $\mathrm{CW}$-complexes is an $\mathcal{A}$-localization if the induced homomorphisms of the homotopy and (reduced integral) homology groups are $\mathcal{A}$-localizations.

Let $G$ be a group, let $\alpha: L \longrightarrow M$ be a surjective combinatorial map of a CW-complex $L$ and a finite simplicial complex $M$ and let $n$ be a positive integer such that $\tilde{H}_{i}\left(\alpha^{-1}(\Delta) ; G\right)=0$ for every $i<n$ and every simplex $\Delta$ of $M$. One can show by induction on the number of simplexes of $M$ using the Mayer-Vietoris sequence and the Five Lemma that $\alpha_{*}: \tilde{H}_{i}(L ; G) \longrightarrow \tilde{H}_{i}(M ; G)$ is an isomorphism for $i<n$. We will refer to this fact as the combinatorial Vietoris-Begle theorem. 
Proposition 2.1 Let $m \geq k+2, k \geq 2$ and let $M$ be an $(m-1)$-connected finite simplicial complex. Let $\omega: E W(M, k) \longrightarrow M$ be the standard resolution for a cellular map $f: N \longrightarrow K(G, k)$ from a subcomplex $N$ of $M$ containing $M^{[k]}$. Then $E W(M, k)$ is $(k-1)$-connected and for every $1 \leq i \leq m-2$, $\pi_{i}(E W(M, k))$ is

(i) $p$-torsion if $G=\mathbf{Z}_{p}$;

(ii) $p$-torsion and $\pi_{k}(E W(M, k))$ is $p$-divisible if $G=\mathbf{Z}_{p^{\infty}}$;

(iii) $p$-local if $G=\mathbf{Z}_{(p)}$ and $\emptyset$-local if $G=\mathbf{Q}$.

Proof Since $M$ and $K(G, k)$ are $(k-1)$-connected then so is $E W(M, k)$. Recall that $\omega$ is a surjective combinatorial map and for every simplex $\Delta$ of $M$, $\omega^{-1}(\Delta)$ is either contractible or homotopy equivalent to $K(G, k)$.

(i) By the generalized Hurewicz theorem the groups $H_{i}\left(K\left(\mathbf{Z}_{p}, k\right)\right), i \geq 1$ are $p$-torsion. Then $H_{i}\left(K\left(\mathbf{Z}_{p}, k\right)\right), i \geq 1$ is $p$-local and $H_{i}\left(K\left(\mathbf{Z}_{p}, k\right) ; \mathbf{Q}\right)=$ $H_{i}\left(K\left(\mathbf{Z}_{p}, k\right)\right) \otimes \mathbf{Q}=0, i \geq 1$. Let $q \in \mathcal{P}$ and $q \neq p$. The $p$-locality of $H_{i}\left(K\left(\mathbf{Z}_{p}, k\right)\right), i \geq 1$ implies that $H_{i}\left(K\left(\mathbf{Z}_{p}, k\right) ; \mathbf{Z}_{q}\right)=0, i \geq 1$. Then, since $M$ is $(m-1)$-connected, by the combinatorial Vietoris-Begle theorem we get that $H_{i}\left(E W(M, k) ; \mathbf{Z}_{q}\right)=0$ and $H_{i}(E W(M, k) ; \mathbf{Q})=0,1 \leq i \leq m-1$. From the universal coefficient theorem it follows that the last conditions imply that $H_{i}(E W(M, k)) \otimes \mathbf{Q}=0$ for $1 \leq i \leq m-1$ and $H_{i}(E W(M, k)) * \mathbf{Z}_{q}=0$ for $1 \leq$ $i \leq m-2$. Hence $H_{i}(E W(M, k))$ is torsion and $q$-torsion free for $1 \leq i \leq m-2$ and every $q \in \mathcal{P}, q \neq p$. Therefore $H_{i}(E W(M, k)), 1 \leq i \leq m-2$ is $p$-torsion and by the generalized Hurewicz theorem $\pi_{i}(E W(M, k)), 1 \leq i \leq m-2$ is $p$-torsion.

(ii) Note that the proof of (i) applies not only for $G=\mathbf{Z}_{p}$ but also for $G=\mathbf{Z}_{p^{\infty}}$. Therefore we can conclude that $\pi_{i}(E W(M, k))$ is $p$-torsion for $1 \leq i \leq m-2$.

By the Hurewicz theorem $\pi_{k}(E W(M, k))=H_{k}(E W(M, k))$. To show that $H_{k}(E W(M, k))$ is $p$-divisible first observe that $H_{k}\left(K\left(\mathbf{Z}_{p^{\infty}}, k\right)\right)=\mathbf{Z}_{p^{\infty}}$ and by the universal coefficient theorem $H_{k}\left(K\left(\mathbf{Z}_{p \infty}, k\right) ; \mathbf{Z}_{p}\right)=\mathbf{Z}_{p^{\infty}} \otimes \mathbf{Z}_{p}=0$. Then since $M$ is $k$-connected the combinatorial Vietoris-Begle theorem implies that $H_{k}\left(E M(M, k) ; \mathbf{Z}_{p}\right)=0$. Once again by the universal coefficient theorem $H_{k}(E W(M, k)) \otimes \mathbf{Z}_{p}=0$ and therefore $H_{k}(E W(M, k))$ is $p$-divisible.

(iii) We will prove the case $G=\mathbf{Z}_{(p)}$. The case $G=\mathbf{Q}$ is similar to $G=$ $\mathbf{Z}_{(p)}$. The proof applies well-known results of Rational Homotopy Theory [11]. 
The $p$-locality of $\pi_{i}\left(K\left(\mathbf{Z}_{(p)}, k\right)\right)$ implies that $H_{i}\left(K\left(\mathbf{Z}_{(p)}, k\right)\right), i \geq 1$ are $p$ local. Then by the reasoning based on the combinatorial Vietoris-Begle and universal coefficient theorems that we used in the proof of (i) one can show that $H_{i}(E W(M, k)), 1 \leq i \leq m-2$ are $p$-local and $H_{m-1}(E W(M, k))$ is $q$-divisible for every prime $q \neq p$. Let a map $\alpha: E W(M, k) \longrightarrow L$ be a $p$ localization of $E W(M, k)$. Then $\alpha$ induces an isomorphism of $H_{i}(E W(M, k))$ and $H_{i}(L)$ for $1 \leq i \leq m-2$ and an epimorphism of $H_{m-1}(E W(M, k))$ and $H_{m-1}(L)$. Hence by the Whitehead theorem the groups $\pi_{i}(E W(M, k))$ and $\pi_{i}(L)$ are isomorphic for $1 \leq i \leq m-2$. Thus $\pi_{i}(E W(M, k)), 1 \leq i \leq m-2$ is $p$-local.

The following proposition is an infinite dimensional version of the implication $(3) \Rightarrow(1)$ of Theorem 1.7.

Proposition 2.2 Let $K$ be a simply connected $C W$-complex such that $K$ has only finitely many non-trivial homotopy groups. Let $X$ be a compactum such that $\operatorname{dim}_{\pi_{i}(K)} X \leq i$ for $i>1$. Then e-dim $X \leq K$.

Proof Let $n$ be such that $\pi_{i}(K)=0$ for $i \geq n$ and let $\psi: A \longrightarrow K$ be a map from a closed subset $A$ of $X$ into $K$. Represent $X$ as the inverse limit $X=\lim _{\leftarrow} K_{j}$ of finite simplicial complexes $K_{j}$ with combinatorial bonding maps $h_{l}^{j}: K_{l} \longrightarrow K_{j}, l>j$ and the projections $p_{j}: X \longrightarrow K_{j}$ such that $\operatorname{diam} p_{j}^{-1}(\Delta) \leq 1 / j$ for every simplex $\Delta$ of $K_{j}$. Let $j$ be so large that there is a map $f: N \longrightarrow K$ from a subcomplex $N$ of $K_{j}$ such that $A \subset p_{j}^{-1}(N)$ and $\left.f \circ p_{j}\right|_{A}$ is homotopic to $\psi$. Then, since $\check{H}^{i+1}\left(X, p_{j}^{-1}(N) ; \pi_{i}(K)\right)=0$ for every $i$, by Obstruction Theory there is a sufficiently large $l>j$ such that $\left.f \circ h_{l}^{j}\right|_{\left(h_{l}^{j}\right)^{-1}(N)}$ extends over the $n$-skeleton of $K_{l}$ and since $\pi_{i}(K)=0$ for $i \geq n$ the map $\left.f \circ h_{l}^{j}\right|_{\left(h_{l}^{j}\right)^{-1}(N)}$ will also extend over $K_{l}$ to $f^{\prime}: K_{l} \longrightarrow K$. Then $\left.f^{\prime} \circ p_{l}\right|_{A}$ is homotopic to $\psi$ and hence $\psi$ extends over $X$. Thus e-dim $X \leq K$.

Let $K^{\prime}$ be a simplicial complex. We say that maps $h: K \longrightarrow K^{\prime}, g: L \longrightarrow L^{\prime}$, $\alpha: L \longrightarrow K$ and $\alpha^{\prime}: L^{\prime} \longrightarrow K^{\prime}$

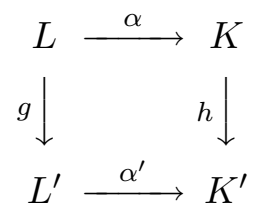

combinatorially commute if for every simplex $\Delta$ of $K^{\prime}$ we have that $\left(\alpha^{\prime} \circ\right.$ $g)\left((h \circ \alpha)^{-1}(\Delta)\right) \subset \Delta$. (The direction in which we want the maps $h, g, \alpha$ and $\alpha^{\prime}$ to combinatorially commute is indicated by the first map in the list. 
Thus saying that $\alpha^{\prime}, h, g$ and $\alpha$ combinatorially commute we would mean that $(h \circ \alpha)\left(\left(\alpha^{\prime} \circ g\right)^{-1}(\Delta)\right) \subset \Delta$ for every simplex $\Delta$ of $K^{\prime}$.) A map $h^{\prime}: K \longrightarrow L^{\prime}$ is said to be a combinatorial lifting of $h$ to $L^{\prime}$ if for every simplex $\Delta$ of $K^{\prime}$ we have that $\left(\alpha^{\prime} \circ h^{\prime}\right)\left(h^{-1}(\Delta)\right) \subset \Delta$.

For a simplicial complex $K$ and $a \in K$, st(a) denotes the union of all the simplexes of $K$ containing $a$. The following proposition whose proof is left to the reader is a collection of simple combinatorial properties of maps.

\section{Proposition 2.3}

(i) Let a compactum $X$ be represented as the inverse limit $X=\lim _{\leftarrow} K_{i}$ of finite simplicial complexes $K_{i}$ with bonding maps $h_{j}^{i}: K_{j} \longrightarrow K_{i}$. Fix $i$ and let $\omega: E W\left(K_{i}, k\right) \longrightarrow K_{i}$ be a resolution of $K_{i}$ which is suitable for $X$. Then there is a sufficiently large $j$ such that $h_{j}^{i}$ admits a combinatorial lifting to $E W\left(K_{i}, k\right)$.

(ii) Let $h: K \longrightarrow K^{\prime}, h^{\prime}: K \longrightarrow L^{\prime}$ and $\alpha^{\prime}: L^{\prime} \longrightarrow K^{\prime}$ be maps of a simplicial complex $K^{\prime}$ and $C W$-complexes $K$ and $L^{\prime}$ such that $h$ and $\alpha^{\prime}$ are combinatorial and $h^{\prime}$ is a combinatorial lifting of $h$. Then there is a cellular approximation of $h^{\prime}$ which is also a combinatorial lifting of $h$.

(iii) Let $K$ and $K^{\prime}$ be simplicial complexes, let maps $h: K \longrightarrow K^{\prime}, g: L \longrightarrow$ $L^{\prime}, \alpha: L \longrightarrow K$ and $\alpha^{\prime}: L^{\prime} \longrightarrow K^{\prime}$ combinatorially commute and let $h$ be combinatorial. Then

$g\left(\alpha^{-1}(s t(x))\right) \subset \alpha^{\prime-1}(s t(h(x)))$ and $h(s t(\alpha(z))) \subset \operatorname{st}\left(\left(\alpha^{\prime} \circ g\right)(z)\right)$

for every $x \in K$ and $z \in L$.

We end this section with recalling basic facts of Bockstein Theory. The Bockstein basis is the following collection of groups $\sigma=\left\{\mathbf{Q}, \mathbf{Z}_{p}, \mathbf{Z}_{p \infty}, \mathbf{Z}_{(p)}: p \in \mathcal{P}\right\}$. For an abelian group $G$ the Bockstein basis $\sigma(G)$ of $G$ is a subcollection of $\sigma$ defined as follows:

$\mathbf{Z}_{(p)} \in \sigma(G)$ if $G / \operatorname{Tor} G$ is not divisible by $p$;

$\mathbf{Z}_{p} \in \sigma(G)$ if $\operatorname{Tor}_{p} G$ is not divisible by $p$;

$\mathbf{Z}_{p \infty} \in \sigma(G)$ if $\operatorname{Tor}_{p} G \neq 0$ and $\operatorname{Tor}_{p} G$ is divisible by $p$;

$\mathbf{Q} \in \sigma(G)$ if $G / \operatorname{Tor} G \neq 0$ and $G / \operatorname{Tor} G$ is divisible by every $p \in \mathcal{P}$.

Let $X$ be a compactum. The Bockstein theorem says that

$\operatorname{dim}_{G} X=\sup \left\{\operatorname{dim}_{E} X: E \in \sigma(G)\right\}$. 
The Bockstein inequalities relate the cohomological dimensions for different groups of Bockstein basis. We will use the following inequalities:

$\operatorname{dim}_{\mathbf{Z}_{p} \infty} X \leq \operatorname{dim}_{\mathbf{Z}_{p}} X \leq \operatorname{dim}_{\mathbf{Z}_{p} \infty} X+1$

$\operatorname{dim}_{\mathbf{z}_{p}} X \leq \operatorname{dim}_{\mathbf{z}_{(p)}} X$ and $\operatorname{dim}_{\mathbf{Q}} X \leq \operatorname{dim}_{\mathbf{Z}_{(p)}} X$.

Finally recall that $\operatorname{dim}_{G} X \leq \operatorname{dim}_{\mathbf{Z}} X$ for every abelian group $G$.

\section{Proof of Theorem 1.6}

Let $m=n+2$. Represent $X$ as the inverse limit $X=\lim _{\leftarrow}\left(K_{i}, h_{i}\right)$ of finite simplicial complexes $K_{i}$ with combinatorial bonding maps $h_{i+1}: K_{i+1} \longrightarrow K_{i}$ and the projections $p_{i}: X \longrightarrow K_{i}$ such that for every simplex $\Delta$ of $K_{i}$, $\operatorname{diam}\left(p_{i}^{-1}(\Delta)\right) \leq 1 / i$. We will construct by induction finite simplicial complexes $L_{i}$ and maps $g_{i+1}: L_{i+1} \longrightarrow L_{i}, \alpha_{i}: L_{i} \longrightarrow K_{i}$ such that

(a) $L_{i}=K_{i}^{[m]}$ and $\alpha_{i}: L_{i} \longrightarrow K_{i}$ is the inclusion. The simplicial structure of $L_{1}$ is induced from $K_{1}^{[m]}$ and the simplicial structure of $L_{i}, i>1$ is defined as a sufficiently small barycentric subdivision of $K_{i}^{[m]}$. We will refer to this simplicial structure while constructing standard resolutions of $L_{i}$. It is clear that $\alpha_{i}$ is always a combinatorial map;

(b) the maps $h_{i+1}, g_{i+1}, \alpha_{i+1}$ and $\alpha_{i}$ combinatorially commute. Recall that this means that for every simplex $\Delta$ of $K_{i},\left(\alpha_{i} \circ g_{i+1}\right)\left(\left(h_{i+1} \circ \alpha_{i+1}\right)^{-1}(\Delta)\right) \subset \Delta$.

We will construct $L_{i}$ in such a way that $Z=\lim _{\leftarrow}\left(L_{i}, g_{i}\right)$ will be of $\operatorname{dim} \leq n$ and $Z$ will admit a cell-like map onto $X$ satisfying the conclusions of the theorem. Assume that the construction is completed for $i$. We proceed to $i+1$ as follows.

Let $E \in \sigma$ be such that $\operatorname{dim}_{E} X \leq k, 2 \leq k \leq n$ and let $f: N \longrightarrow K(E, k)$ be a cellular map from a subcomplex $N$ of $L_{i}, L_{i}^{[k]} \subset N$. Let $\omega_{L}: E W\left(L_{i}, k\right) \longrightarrow$ $L_{i}$ be the standard resolution of $L_{i}$ for $f$. We are going to construct from $\omega_{L}: E W\left(L_{i}, k\right) \longrightarrow L_{i}$ a resolution $\omega: E W\left(K_{i}, k\right) \longrightarrow K_{i}$ of $K_{i}$ suitable for $X$. If $\operatorname{dim} K_{i} \leq k$ set $\omega=\alpha_{i} \circ \omega_{L}: E W\left(K_{i}, k\right)=E W\left(L_{i}, k\right) \longrightarrow K_{i}$.

If $\operatorname{dim} K_{i}>k$ set $\omega_{k}=\alpha_{i} \circ \omega_{L}: E W_{k}\left(K_{i}, k\right)=E W\left(L_{i}, k\right) \longrightarrow K_{i}$ and we will construct by induction resolutions $\omega_{j}: E W_{j}\left(K_{i}, k\right) \longrightarrow K_{i}, k+1 \leq j \leq \operatorname{dim} K_{i}$ such that $E W_{j}\left(K_{i}, k\right)$ is a subcomplex of $E W_{j+1}\left(K_{i}, k\right)$ and $\omega_{j+1}$ extends $\omega_{j}$ for every $k \leq j<\operatorname{dim} K_{i}$. The construction is carried out as follows.

Assume that $\omega_{j}: E W_{j}\left(K_{i}, k\right) \longrightarrow K_{i}, k \leq j<\operatorname{dim} K_{i}$ is constructed. For every simplex $\Delta$ of $K_{i}$ of $\operatorname{dim}=j+1$ consider the subcomplex $\omega_{j}^{-1}(\Delta)$ of 
$E W_{j}\left(K_{i}, k\right)$. Enlarge $\omega_{j}^{-1}(\Delta)$ by attaching cells of dim $\geq m+1$ in order to get a subcomplex with trivial homotopy groups in $\operatorname{dim} \geq m$. Let $E W_{j+1}\left(K_{i}, k\right)$ be $E W_{j}\left(K_{i}, k\right)$ with all the cells attached for all $(j+1)$-dimensional simplexes $\Delta$ of $K_{i}$ and let $\omega_{j+1}: E W_{j+1}\left(K_{i}, k\right) \longrightarrow K_{i}$ be an extension of $\omega_{j}$ sending the interior points of the attached cells to the interior of the corresponding $\Delta$.

Finally denote $E W\left(K_{i}, k\right)=E W_{j}\left(K_{i}, k\right)$ and $\omega=\omega_{j}: E W_{j}\left(K_{i}, k\right) \longrightarrow K_{i}$ for $j=\operatorname{dim} K_{i}$. Note that since we attach cells only of $\operatorname{dim}>m$, the $m$-skeleton of $E W\left(K_{i}, k\right)$ coincides with the $m$-skeleton of $E W\left(L_{i}, k\right)$.

Let us show that $E W\left(K_{i}, k\right)$ is suitable for $X$. Fix a simplex $\Delta$ of $K_{i}$ and denote $M=\alpha_{i}^{-1}(\Delta)$. First note that $M$ is $(m-1)$-connected, $\omega^{-1}(\Delta)$ is $(k-1)$-connected, $\pi_{j}\left(\omega^{-1}(\Delta)\right)=0$ for $j \geq m$ and $\pi_{j}\left(\omega^{-1}(\Delta)\right)=\pi_{j}\left(\omega_{L}^{-1}(M)\right)$ for $k \leq j \leq n$. Also note that since $\operatorname{dim}_{\mathbf{Z}} X \leq n, \operatorname{dim}_{\pi_{j}\left(\omega^{-1}(\Delta)\right)} X \leq \operatorname{dim}_{\mathbf{Z}} X \leq$ $n$ for $n \leq j$. In order to show that e- $\operatorname{dim} X \leq \omega^{-1}(\Delta)$ consider separately the following cases.

Case $1 E=\mathbf{Z}_{p}$. By (i) of Proposition 2.1, $\pi_{j}\left(\omega^{-1}(\Delta)\right)=\pi_{j}\left(\omega_{L}^{-1}(M)\right), k \leq$ $j \leq n$ is $p$-torsion. Hence by Bockstein Theory $\operatorname{dim}_{\pi_{j}\left(\omega^{-1}(\Delta)\right)} X \leq \operatorname{dim}_{\mathbf{Z}_{p}} X \leq k$ for $k \leq j \leq n$. Therefore by Proposition 2.2, e-dim $X \leq \omega^{-1}(\Delta)$.

Case $2 E=\mathbf{Z}_{p^{\infty}}$. By (ii) of Proposition 2.1, $\pi_{j}\left(\omega^{-1}(\Delta)\right)=\pi_{j}\left(\omega_{L}^{-1}(M)\right)$, $k \leq j \leq n$ is $p$-torsion and $\pi_{k}\left(\omega^{-1}(\Delta)\right)=\pi_{k}\left(\omega_{L}^{-1}(M)\right)$ is $p$-divisible. Hence by the Bockstein theorem and inequalities $\operatorname{dim}_{\pi_{k}\left(\omega^{-1}(\Delta)\right)} X \leq \operatorname{dim}_{\mathbf{Z}_{p} \infty} X \leq k$ and $\operatorname{dim}_{\pi_{j}\left(\omega^{-1}(\Delta)\right)} X \leq \operatorname{dim}_{\mathbf{Z}_{p}} X \leq \operatorname{dim}_{\mathbf{Z}_{p} \infty} X+1 \leq k+1$ for $k+1 \leq j \leq n$. Therefore by Proposition 2.2, e-dim $X \leq \omega^{-1}(\Delta)$.

Case $3 E=\mathbf{Z}_{(p)}$. By (iii) of Proposition 2.1, $\pi_{j}\left(\omega^{-1}(\Delta)\right)=\pi_{j}\left(\omega_{L}^{-1}(M)\right)$, $k \leq j \leq n$ is $p$-local. Then $\sigma\left(\pi_{j}\left(\omega^{-1}(\Delta)\right)\right)$ may possibly contain only the groups $\mathbf{Z}_{p}, \mathbf{Z}_{p^{\infty}}, \mathbf{Z}_{(p)}$ and $\mathbf{Q}$. Hence by the Bockstein theorem and inequalities $\operatorname{dim}_{\pi_{j}\left(\omega^{-1}(\Delta)\right)} X \leq \operatorname{dim}_{\mathbf{Z}_{(p)}} X \leq k$ for every $k \leq j \leq n$. Therefore by Proposition 2.2, e-dim $X \leq \omega^{-1}(\Delta)$.

Case $4 E=\mathbf{Q}$. By (iii) of Proposition 2.1, $\pi_{j}\left(\omega^{-1}(\Delta)\right)=\pi_{j}\left(\omega_{L}^{-1}(M)\right)$, $k \leq j \leq n$ is $\emptyset$-local. Then $\sigma\left(\pi_{j}\left(\omega^{-1}(\Delta)\right)\right)$ may possibly contain only $\mathbf{Q}$ and hence $\operatorname{dim}_{\pi_{j}\left(\omega^{-1}(\Delta)\right)} X \leq \operatorname{dim}_{\mathbf{Q}} X \leq k$ for every $k \leq j \leq n$. Therefore by Proposition 2.2, e-dim $X \leq \omega^{-1}(\Delta)$.

Thus we have shown that $E W\left(K_{i}, k\right)$ is suitable for $X$. Replacing $K_{i+1}$ by $K_{j}$ with a sufficiently large $j$ we may assume by (i) of Proposition 2.3 that there is a combinatorial lifting of $h_{i+1}$ to $h_{i+1}^{\prime}: K_{i+1} \longrightarrow E W\left(K_{i}, k\right)$. By (ii) of Proposition 2.3 we replace $h_{i+1}^{\prime}$ by its cellular approximation preserving the property of $h_{i+1}^{\prime}$ of being a combinatorial lifting of $h_{i+1}$. 
Then $h_{i+1}^{\prime}$ sends the $m$-skeleton of $K_{i+1}$ to the $m$-skeleton of $E W\left(K_{i}, k\right)$. Recall that the $m$-skeleton of $E W\left(K_{i}, k\right)$ is contained in $E W\left(L_{i}, k\right)$ and hence one can define $g_{i+1}=\left.\omega_{L} \circ h_{i+1}^{\prime}\right|_{K_{i+1}^{[m]}}: L_{i+1}=K_{i+1}^{[m]} \longrightarrow L_{i}$. Finally define a simplicial structure on $L_{i+1}$ to be a sufficiently small barycentric subdivision of $K_{i+1}^{[m]}$ such that

(c) $\operatorname{diam} g_{i+1}^{j}(\Delta) \leq 1 / i$ for every simplex $\Delta$ in $L_{i+1}$ and $j \leq i$ where $g_{i}^{j}=$ $g_{j+1} \circ g_{j+2} \circ \ldots \circ g_{i}: L_{i} \longrightarrow L_{j}$.

It is easy to check that the properties (a) and (b) are satisfied.

Denote $Z=\lim _{\leftarrow}\left(L_{i}, g_{i}\right)$ and let $r_{i}: Z \longrightarrow L_{i}$ be the projections. For constructing $L_{i+1}$ we used an arbitrary map $f: N \longrightarrow K(E, k), E \in \sigma$ such that $\operatorname{dim}_{E} X \leq k, 2 \leq k \leq n$ and $N$ is a subcomplex of $L_{i}$ containing $L_{i}^{[k]}$. Let us show that choosing $E \in \sigma$ and $f$ in an appropriate way for each $i$ we can achieve that $\operatorname{dim}_{G} Z \leq k$ for every integer $k$ and group $G$ such that $\operatorname{dim}_{G} X \leq k \geq 2$.

Let $E \in \sigma$ be such that $\operatorname{dim}_{E} X \leq k, 2 \leq k \leq n$ and let $\psi: F \longrightarrow K(E, k)$ be a map of a closed subset $F$ of $L_{j}$. Then by (c) for a sufficiently large $i>j$ the map $\left.\psi \circ g_{i}^{j}\right|_{\left(g_{i}^{j}\right)^{-1}(F)}$ extends over a subcomplex $N$ of $L_{i}$ to a map $f: N \longrightarrow K(E, k)$. Extending $f$ over $L_{i}^{[k]}$ we may assume that $L_{i}^{[k]} \subset N$. Replacing $f$ by its cellular approximation we also assume that $f$ is cellular. Now suppose that we use this map $f$ for constructing $L_{i+1}$.

Since $g_{i+1}$ factors through $E W\left(L_{i}, k\right)$, the map $\left.f \circ g_{i+1}\right|_{g_{i+1}^{-1}(N)}: g_{i+1}^{-1}(N) \longrightarrow$ $K(E, k)$ extends to a map $f^{\prime}: L_{i+1} \longrightarrow K(E, k)$. Then $\left.f^{\prime}\right|_{\left(g_{i+1}^{j}\right)^{-1}(F)}$ is homotopic to $\left.\psi \circ g_{i+1}^{j}\right|_{\left(g_{i+1}^{j}\right)^{-1}(F)}:\left(g_{i+1}^{j}\right)^{-1}(F) \longrightarrow K(E, k)$ and therefore $\left.\psi \circ g_{i+1}^{j}\right|_{\left(g_{i+1}^{j}\right)^{-1}(F)}$ extends over $L_{i+1}$. Now since we need to solve only countably many extension problems for every $L_{j}$ with respect to $K(E, k)$ for every $E \in \sigma$ such that $\operatorname{dim}_{E} X \leq k, 2 \leq k \leq n$ we can choose for each $i$ a map $f: N \longrightarrow K(E, k)$ from a subcomplex $N$ of $L_{i}$ in the way described above to achieve that $\operatorname{dim}_{E} Z \leq k$ for every $E \in \sigma$ such that $\operatorname{dim}_{E} X \leq k, 2 \leq k \leq n$ (an algorithm how to assign extension problems to various indices $i$ can be found in [6] and [9]). Then by Bockstein Theory $\operatorname{dim}_{\mathbf{Z}} Z \leq n$ and $\operatorname{dim}_{G} Z \leq k$ for every $G$ such that $\operatorname{dim}_{G} X \leq k \geq 2$. Since $Z$ is finite dimensional and $\operatorname{dim}_{\mathbf{Z}} Z \leq n$ we get that $\operatorname{dim} Z \leq n$.

By (iii) of Proposition 2.3, the properties (a) and (b) imply that for every $x \in X$ and $z \in Z$ the following holds:

(d1) $g_{i+1}\left(\alpha_{i+1}^{-1}\left(s t\left(p_{i+1}(x)\right)\right)\right) \subset \alpha_{i}^{-1}\left(s t\left(p_{i}(x)\right)\right)$ and 
(d2) $h_{i+1}\left(s t\left(\left(\alpha_{i+1} \circ r_{i+1}\right)(z)\right)\right) \subset \operatorname{st}\left(\left(\alpha_{i} \circ r_{i}\right)(z)\right)$.

Define a map $r: Z \longrightarrow X$ by $r(z)=\cap\left\{p_{i}^{-1}\left(s t\left(\left(\alpha_{i} \circ r_{i}\right)(z)\right)\right): i=1,2, \ldots\right\}$. Then (d2) implies that $r$ is indeed well-defined and continuous.

The properties (d1) and (d2) also imply that for every $x \in X$

$r^{-1}(x)=\lim _{\leftarrow}\left(\alpha_{i}^{-1}\left(s t\left(p_{i}(x)\right)\right),\left.g_{i}\right|_{\alpha_{i}^{-1}\left(s t\left(p_{i}(x)\right)\right)}\right)$

where the map $\left.g_{i}\right|_{\ldots}$ is considered as a map to $\alpha_{i-1}^{-1}\left(s t\left(p_{i-1}(x)\right)\right)$.

Since $r^{-1}(x)$ is not empty for every $x \in X, r$ is a map onto and let us show that $r^{-1}(x)$ is cell-like. Let $\phi: r^{-1}(x) \longrightarrow K$ be a map to a CW-complex $K$. Then since $r^{-1}(x)=\lim _{\leftarrow}\left(\alpha_{i}^{-1}\left(s t\left(p_{i}(x)\right)\right),\left.g_{i}\right|_{\ldots}\right)$ there is a sufficiently large $i$ such that the map $\phi$ can be factored up to homotopy through the map $\gamma=\left.r_{i}\right|_{r^{-1}(x)}$ : $r^{-1}(x) \longrightarrow T=\alpha_{i}^{-1}\left(s t\left(p_{i}(x)\right)\right)$, that is there is a map $\beta: T \longrightarrow K$ such that that $\phi$ is homotopic to $\beta \circ \gamma$. Note that $s t\left(p_{i}(x)\right)$ is contractible and $T$ is homeomorphic to the $m$-skeleton of $s t\left(p_{i}(x)\right)$. Hence $T$ is $(m-1)$-connected and since $r^{-1}(x)$ is of $\operatorname{dim} \leq n=m-2$, the map $\gamma$ is null-homotopic. Then $\phi$ is also null-homotopic and hence $r$ is a cell-like map. The theorem is proved.

\section{References}

[1] R. J. Daverman, Hereditarily aspherical compacta and cell-like maps. Topology Appl. 41 (1991), no. 3, 247-254.

[2] A. N. Dranishnikov, On a problem of P. S. Aleksandrov. (Russian) Mat. Sb. (N.S.) 135(177) (1988), no. 4, 551-557, 560; translation in Math. USSR-Sb. 63 (1989), no. 2, 539-545.

[3] A. N. Dranishnikov, On homological dimension modulo $p$. (Russian) Mat. Sb. (N.S.) 132(174) (1987), no. 3, 420-433, 446; translation in Math. USSR-Sb. 60 (1988), no. 2, 413-425.

[4] A. N. Dranishnikov, $K$-theory of Eilenberg-Mac Lane spaces and cell-like mapping problem. Trans. Amer. Math. Soc. 335 (1993), no. 1, 91-103.

[5] A. N. Dranishnikov, Extension of mappings into $C W$-complexes. (Russian) Mat. Sb. 182 (1991), no. 9, 1300-1310; translation in Math. USSR-Sb. 74 (1993), no. $1,47-56$

[6] A. Dranishnikov, Cohomological dimension theory of compact metric spaces. Topology Atlas Invited Contributions, http://at.yorku.ca/topology/taic.htm

[7] A. Dranishnikov, J. Dydak, Extension dimension and extension types. Tr. Mat. Inst. Steklova 212 (1996), Otobrazh. i Razmer., 61-94; translation in Proc. Steklov Inst. Math. 1996, no. 1 (212), 55-88 
[8] Alexander Dranishnikov, Jerzy Dydak, Extension theory of separable metrizable spaces with applications to dimension theory. Trans. Amer. Math. Soc. 353 (2001), no. 1, 133-156.

[9] Alexander N. Dranishnikov, Dušan Repovš, On Alexandroff theorem for general abelian groups. Topology Appl. 111 (2001), no. 3, 343-353.

[10] R. D. Edwards, A theorem and a question related to cohomological dimension and cell-like maps. Notices of the AMS, 25(1978), A-259.

[11] Yves Felix, Stephen Halperin, Jean-Claude Thomas, Rational homotopy theory. Graduate texts in mathematics no. 205, New York, Springer-Verlag, 2001.

[12] Jerzy Dydak, John J. Walsh, Infinite-dimensional compacta having cohomological dimension two: an application of the Sullivan conjecture. Topology 32 (1993), no. 1, 93-104.

[13] Akira Koyama, Katsuya Yokoi, On Dranishnikov's cell-like resolution. Geometric topology: Dubrovnik 1998. Topology Appl. 113 (2001), no. 1-3, 87-106.

[14] Michael Levin, Constructing compacta of different extensional dimensions. Canad. Math. Bull. 44(2001), no. 1, 80-86.

[15] Michael Levin, Acyclic resolutions for arbitrary groups. Israel J. Math. 135 (2003), 193-203.

[16] John J. Walsh, Dimension, cohomological dimension, and cell-like mappings. Shape theory and geometric topology (Dubrovnik, 1981), pp. 105-118, Lecture Notes in Math., 870, Springer, Berlin-New York, 1981.

Department of Mathematics

Ben Gurion University of the Negev

P.O.B. 653

Be'er Sheva 84105, ISRAEL

Email: mlevine@math.bgu.ac.il

Received: 7 April 2003 Revised: 1 December 2003 